\title{
Gut, aber nicht gut genug
}

\section{Die Reform der Pflegeversicherung verbessert die Situation für pflegebedürftige Menschen - doch viele Widersprüche und Ungereimtheiten bleiben}

\author{
Ingrid Hastedt
}

Ingrid Hastedt ist Vorsitzende des

Vorstandes des Wohlfahrtswerkes für Baden-Württemberg. Die Organisation wurde im Jahre 1817 gegründet und bietet heute als privatrechtliche Stiftung zahlreiche soziale Dienst und Einrichtungen insbesondere für ältere Menschen an.

Internet http://www.wohlfahrtswerk.de
Das Gesetz zur strukturellen Weiterentwicklung der Pflegeversicherung ist am 1. Juli 2008 in Kraft getreten. Die ersten Bewertungen aus der Praxis bestätigen die im Gesetzgebungsverfabren geäußerten Einschätzungen: Die Reform der Pflegeversicherung bringt zwar Fortschritte, lässt aber auch viele Fragen unbeantwortet.

Die jüngste Reform der Pflegeversicherung war ein Vorhaben, das die Große Koalition im Koalitionsvertrag von 2005 festgeschrieben hat. Wesentliche Ziele waren eine Verbesserung der Einnahmen der Pflegekassen und auf der Ausgabenseite Leistungsverbesserungen für Menschen mit Demenz und ein Inflationsausgleich für alle Leistungsbezieher. In den Medien wurde die Nachhaltigkeit der Einnahmen diskutiert und kurz vor der Verabschiedung entspann sich eine lebhafte öffentliche Diskussion zu »Pflegestützpunkten ", einer von der Bundesgesundheitsministerin eingebrachten Idee. Das »Gesetz zur strukturellen Weiterentwicklung der Pflegeversicherung « (PfWG) enthält jedoch darüber hinaus diverse Änderungen mit Relevanz für Anbieter und Nutzer, über die hier ein Überblick gegeben werden soll.

\section{Geringfügige strukturelle Verbesserungen}

Unverändert bleiben die meisten Strukturen der Leistungen für Pflegebedürftige:

- sektorale Differenzierung der Versicherungsleistungen nach Inanspruchnahme von ambulanten, teilstationären und stationären Angeboten

- reine Sachleistung bei stationärer Pflege, sonst alternativ Sach- oder Geldleistungen mit Möglichkeit, diese zu kombinieren

- Zuzahlungsprinzip im Teilkasko-Sinn Ein Schritt hin zu weniger Bürokratie und zu Synergieeffekten zwischen ambulantem, teilstationärem und stationärem
Pflegesektor ist die Einführung eines Gesamtversorgungsvertrags für Träger: Ambulanten, teilstationären und stationären Einrichtungen wird damit bei örtlichem und organisatorischem Verbund eine gemeinsame Zulassung bei den Pflegekassen ermöglicht.

Der Grundsatz "ambulant vor stationär « wird ernster genommen als bisher: Die finanzielle Benachteiligung ambulant versorgter Personen wird schrittweise reduziert durch Anheben der an diese ausgezahlten Leistungsbeträge (siehe Tabellen 1 und 2 auf der nächsten Seite). Dennoch übersteigt in der Endausbaustufe ab 1. Januar 2012 der eingefrorene Sachleistungswert für Pflegeheimbewohner (1) die ambulanten Sachleistungen in Pflegestufe 1 um 573 Euro und in Stufe 2 um 450 Euro. Die seit jeher vorhandene Gleichstellung in Pflegestufe 3 bleibt unangetastet.

Der Umfang der Pflegebedürftigkeit führt zwar durch steigende Pflegestufe zu steigender Kassenleistung. Ansonsten ist aber - mit Ausnahme der Pflegestufe 3 nicht der Hilfebedarf, sondern die gewählte Versorgungsform ausschlaggebend für die Höhe der Kassenleistung. Von einer echten Umsetzung des Anspruchs »ambulant vor stationär « hätte man erst bei deutlich höheren Anhebungen der ambulanten Leistungsbeträge oder bei Absenkung der stationären Sachleistungen reden können.

Doch weitere Maßnahmen, die jede für sich betrachtet nicht so viel Wirkung haben mag, führen insgesamt zu einer Stärkung der ambulanten Versorgungsform:

\section{Mehr Geld für Tagespflegegäste gibt es nur als Sachleistung}

Eine strukturell bedeutende Neuerung betrifft Tagespflegegäste. Die Tagespflege entlastet pflegende Angehörige und bietet Demenzkranken eine Tagesstruktur. Doch die Nutzer benötigen häufig 


\begin{tabular}{|l|l|l|l|l|}
\hline $\begin{array}{l}\text { Pflege- } \\
\text { stufe }\end{array}$ & $\begin{array}{l}\text { Pflegesach- } \\
\text { leistung } \\
\text { bisher }\end{array}$ & $\begin{array}{l}\text { Pflegesach- } \\
\text { leistung } \\
\text { ab 01.07.2008 }\end{array}$ & $\begin{array}{l}\text { Pflegesach- } \\
\text { leistung } \\
\text { ab 01.01.2010 }\end{array}$ & $\begin{array}{l}\text { Pflegesach- } \\
\text { leistung } \\
\text { ab 01.01.2012 }\end{array}$ \\
\hline $\mathbf{1}$ & $384 €$ & $420 €$ & $440 €$ & $450 €$ \\
\hline $\mathbf{2}$ & $921 €$ & $980 €$ & $1.040 €$ & $1.100 €$ \\
\hline $\mathbf{3}$ & $1.432 €$ & $1.470 €$ & $1.510 €$ & $1.550 €$ \\
\hline
\end{tabular}

Tabelle 1: Ambulante Sachleistungen vor und nach der Reform

\begin{tabular}{|l|c|c|c|c|}
\hline $\begin{array}{l}\text { Pflege- } \\
\text { stufe }\end{array}$ & $\begin{array}{l}\text { Pflegegeld } \\
\text { bisher }\end{array}$ & $\begin{array}{l}\text { Pflegegeld } \\
\text { ab 01.07.2008 }\end{array}$ & $\begin{array}{l}\text { Pflegegeld } \\
\text { ab 01.01.2010 }\end{array}$ & $\begin{array}{l}\text { Pflegegeld } \\
\text { ab 01.01.2012 }\end{array}$ \\
\hline $\mathbf{1}$ & $205 €$ & $215 €$ & $225 €$ & $235 €$ \\
\hline $\mathbf{2}$ & $410 €$ & $420 €$ & $430 €$ & $440 €$ \\
\hline $\mathbf{3}$ & $665 €$ & $675 €$ & $685 €$ & $700 €$ \\
\hline
\end{tabular}

Tabelle 2: Ambulante Geldleistungen vor und nach der Reform

außerdem häusliche Pflege durch einen Pflegedienst. Die Reform hebt die konkurrierende Mittelverwendung des SGB XI-Sachleistungsbudgets teilweise auf: Zusätzlich zu ambulanten Sach- oder Geldleistungen kann der Tagespflegebesuch als Sachleistung abgerechnet werden. Nachdem Tagespflegeeinrichtungen vielerorts über Auslastungsprobleme klagen, die auch zu Schließungen führten, zielt der Gesetzgeber auf eine höhere Preisakzeptanz für Tagespflege. Bemerkenswert ist, dass der Wert der Sachleistung für Tagespflegegäste der Pflegestufe 2 und 3, die ihre Ansprüche maximal ausschöpfen, jenen für Pflegeheimbewohner deutlich übersteigt (siehe Tabelle 3).

Eine weitere Stärkung der häuslichen Pflegesituation soll eine Pflegezeit für berufstätige Angehörige bringen. Um Überforderung von Pflegepersonen vorzubeugen, werden Anreize zum weiteren Ausbau von niederschwelligen Betreuungsangeboten beibehalten und das Budget von 20 auf 50 Millionen Euro aufgestockt. Inwieweit dies ausgeschöpft wird, bleibt abzuwarten.

In der eigenen Häuslichkeit wohnende Tagespflegegäste profitieren bei Verzicht auf Geldleistung am meisten von der Pflegereform:

\begin{tabular}{|l|c|c|c|c|}
\hline $\begin{array}{l}\text { Pflege- } \\
\text { stufe }\end{array}$ & $\begin{array}{l}\text { Pflegegeld } \\
\mathbf{0} \%\end{array}$ & $\begin{array}{l}\text { Pflegesach- } \\
\text { leistung } \\
\mathbf{1 0 0 \%}\end{array}$ & $\begin{array}{l}\text { Sachleistung für } \\
\text { Tagespflege }\end{array}$ & $\begin{array}{l}\text { Wert der } \\
\text { gesamten Pflege- } \\
\text { kassenleistung }\end{array}$ \\
\hline $\mathbf{1}$ & $0 €$ & $420 €$ & $210 €$ & $630 €$ \\
\hline $\mathbf{2}$ & $0 €$ & $980 €$ & $490 €$ & $1.470 €$ \\
\hline $\mathbf{3}$ & $0 €$ & $1.470 €$ & $735 €$ & $2.205 €$ \\
\hline
\end{tabular}

Tabelle 3: Sachleistungen für ambulante Dienste schmälern das Budget für Tagespflegegäste nicht mehr.
Artikel 6 des Gesetzes zur strukturellen Weiterentwicklung der Pflegeversicherung (4) ergeben: Im Rahmen von Modellvorhaben können Alten- und Krankenpflegefachkräfte eine Verordnungsermächtigung für Verbands- und Pflegehilfsmittel erhalten und ihnen können auch ärztliche Tätigkeiten, für die sie nach ihrer Ausbildung qualifiziert sind, übertragen werden. Die berufspolitische Diskussion erhält dadurch eine neue Dynamik.

Wegen der leistungsrechtlichen Trennung wurden seit Jahren die fehlenden finanziellen Anreize für Krankenkassen moniert, die Rehabilitation für pflegebedürftige Menschen stärker zu forcieren. Mit einer Maluszahlung von 1.536 Euro (5) falls sechs Monate nach Antragstellung notwendige Rehaleistungen nicht erbracht wurden, wird dies erstmals durchbrochen. Auch das Gutachten des Medizinischen Dienstes (MDK) zur Bewertung der Pflegebedürftigkeit muss nun nicht nur Reha-Potenziale, sondern auch Prävention bewerten und Maßnahmen empfehlen.

Prävention und Rehabilitation sollen zudem durch individuelle Beratung gestärkt werden: Ab 1. Januar 2009 besteht Anspruch auf Pflegeberatung $(\mathbb{S} 7 a)$. Deren Inhalt geht deutlich über die seither in $\mathbb{} 7$ Abs. 2 SGB XI enthaltene Vorgabe hinaus. Während bisher die Leistungen und Hilfen von Anbietern Beratungsinhalt sein sollten, sind nun ausdrücklich auch präventive und rehabilitative Hilfen sowie soziale Hilfe mitgemeint. Die Beratung soll auf Wunsch sogar in der häuslichen Umgebung erfolgen. Wie umfassend dieser Ansatz tatsächlich umsetzbar sein wird, muss sich noch erweisen. Denn die bei den Pflegekassen angesiedelte Pflegeberatung soll von Pflegestützpunkten nach $\$ 92 c$ erbracht werden. Da diese nicht flächendeckend installiert werden und lediglich eine Anschubfinanzierung vom Bund gewährt wird, sind die Erwartungen des Gesetzgebers hoch und vor allem ein Signal für die Bedeutung der fachlichen Begleitung betroffener Menschen und ihren Angehörigen. Dass Antragsteller künftig längstens fünf Wochen auf die Entscheidung der Pflegekasse zur Pflegeeinstufung warten sollen ( $\mathbb{1 8}$ Abs. 3), kann als Symbol für Kundenfreundlichkeit betrachtet werden. 
Wird Pflegesachleistung zu 100 Prozent für einen ambulanten Pflegedienst verwendet, können Versicherte zusätzlich Tagespflegebesuche mit der Pflegekasse abrechnen. Die nachfolgende Tabelle zeigt an einem konkreten Beispiel, bei wie viel Besuchstagen der Anspruch an die Pflegekasse maximal ausgeschöpft wird. Bei Pflegestufe 1 ist beispielsweise für sechs Besuchstage das Pflegeentgelt der Tagespflege durch die Pflegekasse finanziert. Der Eigenanteil für die Gebäudekosten und Verpflegung, die grundsätzlich nicht von der Pflegekasse finanziert werden können, beträgt für diese sechs Tage 75,66 Euro.

\begin{tabular}{|l|l|l|l|l|l|l|}
\hline A & B & C & D & E & F \\
\hline $\begin{array}{l}\text { Pflege- } \\
\text { stufe }\end{array}$ & $\begin{array}{l}\text { Entgelt je Tag } \\
\text { für Pflege }\end{array}$ & $\begin{array}{l}\text { aus Pflegesach- } \\
\text { leistung finanzier- } \\
\text { bare Besuchstage }\end{array}$ & $\begin{array}{l}\text { Besuchstage } \mathbf{x} \\
\text { Pflegeentgelt }\end{array}$ & $\begin{array}{l}\text { Entgelt/Tag } \\
\text { Unterkunft und Her- } \\
\text { kunft zzgl. Investi- } \\
\text { tionskosten zzgl. - } \\
\text { Ausbildungsumlage }\end{array}$ & $\begin{array}{l}\text { Eigenanteil lt. } \\
\text { Spalte E bei } \\
\text { Anzahl Tage } \\
\text { lt. Spalte C }\end{array}$ & $\begin{array}{l}\text { Eigenanteil für } \\
\text { Tagespflegebesuch } \\
\text { in \% }\end{array}$ \\
\hline 1 & $31,66 €$ & 6 Tage & $189,96 €$ & $\begin{array}{l}6,34+5,27+ \\
1 €=12,61 €\end{array}$ & $75,66 €$ & $28,5 \%$ \\
\hline 3 & $45,26 €$ & 10 Tage & $452,60 €$ & $12,61 €$ & $126,10 €$ & $16 \%, 8,93 \%$ \\
\hline
\end{tabular}

Tabelle 4: Beispielsrechnung für die Kombination von ambulanter Pflege und Tagespflege

\section{Qualität kostet Geld}

Mit der Pflegereform wird anerkannt, dass für eine gute Qualität der Versorgung von Pflegeheimbewohnern externe Akteure eine Rolle spielen können: Folgen einer strukturell bedingt schlechten hausärztlichen Versorgung können jetzt vermieden werden durch Beschäftigung eines Heimarztes. (6) Auch die Bedeutung ehrenamtlichen Engagements - samt der Notwendigkeit dies zu organisieren wird erstmals gewürdigt: $\mathbb{\int} 82 \mathrm{~b}$ SGB XI bietet Pflegeheimen die Möglichkeit eines Zuschlags zur Pflegevergütung für diesbezüglichen Organisationsaufwand. Ein weiterer Mentalitätswandel zeigt sich in einer Bonuszahlung von 1.536 Euro gemäß $\int 87 a$ Abs. 4 SGB XI. Damit sollen finanzielle Einbußen von Pflegeheimen gemildert werden, wenn durch aktivierende und rehabilitative Pflege im Heim eine Abstufung in eine niedere Pflegestufe erfolgt.

\section{Transparenz angestrebt}

Das seit Beginn in der Pflegeversicherung enthaltene Bekenntnis zum Markt bleibt unangetastet. Grundvoraussetzungen dafür sind Markttransparenz und Verbraucherschutz. Das Interesse von Verbraucherseite und Medien konzentriert sich momentan darauf, schwarze Schafe zu identifizieren. Das Gesetz zur strukturellen Weiterentwicklung der Pflegeversicherung verankert deshalb die Veröffentlichung von Er- gebnissen der Qualitätsprüfungen des Medizinischen Dienstes (7). Flankierend werden maximal dreijährige Abstände von Qualitätsprüfungen des Medizinischen Dienstes festgeschrieben. (8) Die bisher unter Experten umstrittene Aussagekraft der MDK-Qualitätsbewertung soll durch neue Richtlinien, bei deren Erarbeitung verschiedene Interessenvertretungen $\mathrm{zu}$ beteiligen sind, behoben werden.

\section{Das Umsetzen des »Poolens « ist unklar}

Reagiert wird mit dem neuen Gesetz zur strukturellen Weiterentwicklung der Pflegeversicherung auch auf eine erst seit der vorangegangenen Reform aus dem Jahre 2002 in Fahrt gekommene Debatte um ambulant betreute Wohngemeinschaften. Deren Bewohner sollen mehr Synergieeffekte des gemeinschaftlichen Wohnens nutzen können, indem sie gemeinsam Pflege- und Betreuungsleistungen und hauswirtschaftliche Versorgung als Sachleistung ambulanter Dienste in Anspruch nehmen. Pflegekassen müssen für Betreuungsleistungen, die gemeinschaftlich abgerufen werden, Vergütungen vereinbaren $(\mathbb{S} 89$ Abs. 3). Dieses in \36 genannte »Poolen « macht Betreuungsleistung erstmals sachleistungsfähig. Welche Effekte sich daraus ergeben, hängt allerdings von der faktischen Umsetzung ab, denn der Gesetzestext gibt keine Hinweise zur praktischen Durchführung.

\section{Messen mit zweierlei Maß}

Im häuslichen Bereich zahlt die Pflegekasse deutlich mehr, wenn eine Leistung bei einem Pflegedienst abgerufen wird, als wenn Geldleistung gewählt wird. Die Autonomie der Versicherten wird nun gestärkt: Sollte eine Einzelpflegekraft dem besonderen Wunsch des Pflegebedürftigen besonders entsprechen, kann die Kasse mit dieser Person einen Vertrag ab-

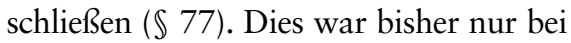
Marktversagen möglich, wenn die Versorgung nicht durch einen zugelassenen Pflegedienst sichergestellt werden kann. Jetzt kann beispielsweise eine Person gleicher Religion oder Kultur gezielt organisiert werden und in Wohngemeinschaften lebende Pflegebedürftige können ausgewählte Personen gemeinsam beauftragen.

Widersprüchlich bleiben dabei die unterschiedlichen Ansprüche an Qualitätssicherung für privat beschaffte Pflegehilfen im Gegensatz zu professionellen Pflegeeinrichtungen. So stärkt die Reform die Position von Einzelpflegekräften, für die keine Qualitätssicherungsvorgaben existieren. Die fachlichen Standards, die von Pflegeeinrichtungen zu erfüllen sind, werden dagegen erneut gesteigert: Neuerdings sind beispielsweise Expertenstandards im Bundesanzeiger zu veröffentlichen und $\mathrm{ab}$ diesem Moment verbindlich. Zwar wird damit klarer, ab wann welcher "Stand des Wissens « gilt. Problematisch ist dabei, dass eine ausdrückliche Finanzierung der Qualitätssicherung, wie sie im SGB V geregelt ist, im Gesetz zur 
strukturellen Weiterentwicklung der Pflegeversicherung unerwähnt und damit weiterhin den Pflegeeinrichtungen versagt bleibt.

\section{Arbeitgeberseite}

Für alle Arbeitgeber in Deutschland steigen aufgrund des Gesetzes zur strukturellen Weiterentwicklung der Pflegeversicherung die Beitragssätze zur Pflegeversicherung. Das Pflegezeitgesetz in Artikel 3 bedeutet für sie organisatorischen Anpassungsbedarf wegen der Einführung einer Pflegezeit für Beschäftigte. dem beide Verhandlungspartner mit deren Anwendung einverstanden sein müssen. Ob Personalaufwand, der durch ortsübliche oder tarifliche Vergütung entsteht, durch Pflegeentgelte damit finanzierbar werden wird, ist offen. Es hieße eine Abkehr von der Praxis der Pflegesatzverhandlungen vergangener Jahre. Diese waren in vielen Regionen reine Preisverhandlungen, von Pflegekassen und Sozialhilfe dominiert und durch fehlende Bezugnahme zwischen Kosten und Entgelt geprägt. Neben diese unabhängig vom Aufwand hergeleiteten Preise setzt das Gesetz zur strukturellen Weiterentwicklung der Pflegeversicherung nun eine Parallelstruktur: Die Finanzie-
Qualitätsprüfungen im Auftrag der Pflegekassen.

(8) $\mathbb{S}$ 114, 114a SGB XI.

(9) Rheinisch-Westfälisches Institut für Wirtschaftsforschung e. V. (Hg.): Pflegeheim Rating Report 2007 - Wachstum und Restrukturierung, Essen, 2007.

(10) Urteil des Bundessozialgerichts vom 14. Dezember 2000, Az: B 3 P 19/00 R; aber auch Urteil des LSG Baden-Württemberg; z. B. das Urteil vom 7. Dezember 2007, in dem die Schiedsstelle BadenWürttemberg vom Landessozialgericht gerügt wird für die Berücksichtigung tariflicher Bindung der Arbeitgeber bei der Festsetzung der Entgelte.

\section{»Hilfebedarf und Versorgungsform bestimmen die Leistungshöhe der Kasse"}

Speziell für Arbeitgeber im Pflegesektor kommen Anforderungen tariflicher Art hinzu: Neu ist, dass der Versorgungsvertrag als Abrechnungsvoraussetzung für Anbieter an die Gewährleistung einer »ortsüblichen Vergütung « für die Mitarbeiterinnen und Mitarbeiter geknüpft wird. Fragwürdig ist dieses Anliegen insofern, als der Bewegungsspielraum für Anbieter, ihr Personal angemessen zu entlohnen, gegen null tendiert. Die faktische Verhandlungsmacht bei Entgeltverhandlungen liegt bei Kassen und Sozialhilfeträgern. Wirtschaftlich bedingte Betriebsschließungen sind daher im ambulanten Bereich nichts Ungewöhnliches und ein aktuelles Gutachten des Rheinisch-Westfälisches Institut für Wirtschaftsforschung e. V. (RWI) erachtet 13 Prozent der Pflegeheime als insolvenzgefährdet. (9) Die Verantwortung für die Umsetzbarkeit ortsüblicher Vergütungen liegt deshalb weniger bei den Anbietern und ihren Tarifpartnern, sondern vielmehr auf politischer Ebene - und bei den Gerichten. Letztere haben seit Dezember 2000 (10) den Marktmechanismus so interpretiert, dass Pflegeheimpreise durch Vergleich mit regionalen Mitbewerbern entstehen sollen. Das Gesetz zur strukturellen Weiterentwicklung der Pflegeversicherung schränkt die Anwendung dieser als »externer Vergleich « benannten Methode jetzt ein, in- rung von Personalschlüsseln für Betreuungsassistenz demenzkranker Heimbewohner gemäß \45a signalisiert, dass Entgelte mit Kosten im Zusammenhang stehen. Gleiches gilt für die neu eingeführten Zuschläge zur Pflegevergütung für die Organisation ehrenamtlicher Unterstützung und für Mitglieder von Selbsthilfegruppen ( $\mathbb{8} 82 \mathrm{~b}$ SGB XI-E). Würde in Entgeltverhandlungen der Aufwand generell eine Rolle spielen, wären diese neuen Regelungen überflüssig.

\section{Anmerkungen}

(1) Die Pflegekasse erstattet bei Pflegeheimaufenthalt 1.023 Euro (Pflegestufe 1) bzw. 1.279 Euro (Pflegestufe 2).

(2) Monatlich werden 100 oder 200 Euro gezahlt für Tages- oder Nachtpflege, Kurzzeitpflege und niederschwellige Dienste.

(3) Siehe $\mathbb{\int} 45$ a SGB XI.

(4) Im SGB V eingefügt wurde $\mathbb{S} 63$ Abs. $3 \mathrm{~b}$ und $3 \mathrm{c}$.

(5) Siehe $\mathbb{} 40$ Abs. 3 SGB XI.

(6) $\mathbb{S} 119 \mathrm{~b}$ SGB V.

(7) Der Medizinische Dienst der Krankenkassen ist a) zuständig für die Gutachten zur Pflegeeinstufung und b) für

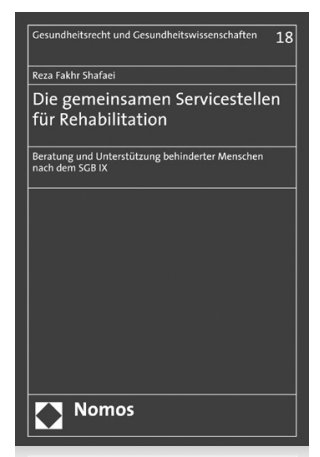

\section{Die gemeinsamen Service- stellen für Rehabilitation}

Beratung und Unterstützung behinderter Menschen nach dem SGB IX

Von RA Dr. Reza Fakhr Shafaei 2008, 276 S., brosch., 58,-€, ISBN 978-3-8329-3207-7 (Gesundheitsrecht und Gesundheitswissenschaften, $B d$. 18)

Das Werk befasst sich mit der Funktion und den Aufgaben der gemeinsamen Servicestellen für Rehabilitation und ordnet sie in das Gefüge des Verwaltungs- und Sozialrechts ein. Der Autor beantwortet die vielfältigen und für die Praxis bedeutsamen Rechtsfragen im Zusammenhang mit der Einführung dieser neuen Institution.

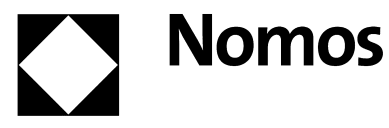

